21st Particles and Nuclei International Conference (PANIC 2017)

International Journal of Modern Physics: Conference Series

Vol. 46 (2018) 1860051 (6 pages)

(C) The Author(s)

DOI: $10.1142 / \mathrm{S} 2010194518600510$

\title{
Propagation of Uncertainties of the Nucleon-Nucleon Potential to the Neutron-Deuteron Elastic Scattering Cross Section
}

\author{
R. Skibiński*, Yu. Volkotrub, J. Golak, K. Topolnicki and H. Witała \\ M. Smoluchowski Institute of Physics, Jagiellonian University, \\ ul.prof.St.Łojasiewicza 11, Kraków, PL-30348, Poland \\ *roman.skibinski@uj.edu.pl
}

Published 3 May 2018

\begin{abstract}
Faddeev formalism is used to study the propagation of theoretical uncertainties from the two-nucleon force to three-nucleon scattering observables. Predictions are obtained with the One-Pion-Exchange Gaussian interaction, for which correlations between its parameters are known. Within the Monte Carlo approach we are able to estimate uncertainties of three-nucleon observables arising from the imprecise knowledge of the OnePion-Exchange Gaussian potential parameters. We found that the uncertainties of this type are small for three-nucleon elastic scattering cross section at investigated here energies up to the nucleon laboratory energy of $200 \mathrm{MeV}$. They remain smaller than the dominant theoretical uncertainty arising from using various models of nucleon-nucleon interactions. We also compare the above-mentioned results with other types of theoretical uncertainties, that is with the ones stemming from order truncation errors and regulator dependencies, present in calculations based on the chiral interaction.
\end{abstract}

Keywords: Nuclear force; three-nucleon system; theoretical uncertainty.

\section{Introduction}

A careful analysis of modern precise nuclear data requires not only the state-ofthe-art theoretical approaches but also estimations of uncertainties of theoretical results. Using in ab-initio calculations nuclear interactions based on different physical assumptions, the uncertainties of potential parameters and, in the case of the chiral forces, the truncation errors related to neglecting the higher orders of chiral expansions as well as the dependence on regulator functions belong to the most important sources of theoretical uncertainties in the few-nucleon sector. In recent years many efforts have been focused on clarifying this situation and on introducing estimators at least for some of the above mentioned uncertainties. The exhaustive

This is an Open Access article published by World Scientific Publishing Company. It is distributed under the terms of the Creative Commons Attribution 4.0 (CC-BY) License. Further distribution of this work is permitted, provided the original work is properly cited. 


\section{R. Skibiński et al.}

discussion of various ways to estimate theoretical uncertainties, mainly in the twonucleon system and in nuclear structure calculations, can be found in a topical issue of J. Phys. G: Nucl. Part. Phys. ${ }^{1}$. In this contribution we apply some of error estimators to the nucleon-deuteron (Nd) elastic scattering cross section.

To this end, we make use of the works of the Granada group focused on revising the existing data base for the nucleon-nucleon (NN) interaction and deriving, among others, the One-Pion-Exchange (OPE) Gaussian NN force ${ }^{2,3}$. The structure of the OPE-Gaussian potential is similar to the structure of the standard semiphenomenological AV18 model ${ }^{4}$, which has been successfully applied to analyze a rich spectrum of nuclear observables, including $\mathrm{Nd}$ scattering, see e.g. Refs. 5 and 6 . In the case of the OPE-Gaussian model the long-range part of the interaction is given by the usual one-pion exchange potential, supplemented by the electromagnetic corrections. The short-range part of the potential is defined by a set of operators accompanied by the sum of Gaussian functions $F_{i, n}(r)$

$$
V_{\text {short }}(\vec{r})=\sum_{n=1}^{18} \hat{O}_{n}\left[\sum_{i=1}^{4} V_{i, n} F_{i, n}(r)\right] \text {. }
$$

The radial Gaussian functions $F_{i, n}(r)$ depend on a free parameter $a_{0}$ defining their widths. This free parameter together with the strength parameters $V_{i, n}$ are fixed from the NN data. The fitting procedure has been done in a careful statistical manner and the " $3 \sigma$ consistent data base" 2 has been used. The resulting values of the $a_{0}$ and $V_{i, n}$ parameters as well as their covariance matrix are presented in Ref. 3 . This can be further used to estimate the uncertainty of three-nucleon (3N) observables arising from the uncertainty of the two-nucleon $(2 \mathrm{~N})$ potential parameters. Here we present the first results of such investigations focusing on the differential cross section for Nd elastic scattering at incoming nucleon laboratory energies $E=65 \mathrm{MeV}$ and $E=200 \mathrm{MeV}$. In addition, these are the first applications of the OPE-Gaussian force to $3 \mathrm{~N}$ reactions, thus the comparison of results with predictions based on the standard potentials, like the AV18, is obviously very interesting.

On the other hand, chiral forces have dominated contemporary studies of fewnucleon processes, see for example Refs. 7-9. It is well known that in the case of the chiral models, the truncation errors as well as the cutoff dependence have to be taken into account while performing the theoretical analysis of few-nucleon processes. A simple prescription to estimate the truncation errors has been proposed in Ref. 10 for the $2 \mathrm{~N}$ system and extended to many-nucleon systems (if many-nucleon forces are neglected) in Ref. 7. Within this method the truncation error $\delta(X)^{(i)}$ of an observable $X$ at $i$-th order of the chiral expansion, with $i=0,2,3, \ldots$, is expressed as

$$
\begin{aligned}
& \delta(X)^{(0)} \geq \max \left(Q^{2}\left|X^{(0)}\right|,\left|X^{(i \geq 0)}-X^{(j \geq 0)}\right|\right) \\
& \delta(X)^{(2)}=\max \left(Q^{3}\left|X^{(0)}\right|, Q\left|\Delta X^{(2)}\right|,\left|X^{(i \geq 2)}-X^{(j \geq 2)}\right|\right) \\
& \delta(X)^{(i)}=\max \left(Q^{i+1}\left|X^{(0)}\right|, Q^{i-1}\left|\Delta X^{(2)}\right|, Q^{i-2}\left|\Delta X^{(3)}\right|\right) \text { for } i \geq 3
\end{aligned}
$$


where $Q$ denotes the chiral expansion parameter, $X^{(i)}$ is a prediction for the observable $X$ at $i$-th order, $\Delta X^{(2)} \equiv X^{(2)}-X^{(0)}$ and $\Delta X^{(i)} \equiv X^{(i)}-X^{(i-1)}$ for $i \geq 3$. Additional conditions: $\delta(X)^{(2)} \geq Q \delta(X)^{(0)}$ and $\delta(X)^{(i)} \geq Q \delta(X)^{(i-1)}$ for $i \geq 3$ are imposed on the truncation errors $\delta(X)^{(i)}$. Such a way of estimation of truncation errors accounts for the fact that the $3 \mathrm{~N}$ force is neglected, which is also the case for the calculations presented here.

We use the same approach and estimate the truncation errors for the cross section obtained with the chiral $\mathrm{NN}$ interaction ${ }^{11}$ at the fifth order of chiral expansion $\left(\mathrm{N}^{4} \mathrm{LO}\right)$. This interaction model uses a local regulator function in coordinate space to avoid ultraviolet divergences. Using various values of the regulator parameter $R$ leads to different predictions for observables. It is expected that the regulator dependence vanishes at a sufficiently high order of chiral expansion. However, in practical calculations performed at $\mathrm{N}^{4} \mathrm{LO}$ the regulator dependence is still visible, increasing the uncertainty of predictions. The spread of predictions obtained with a given model of interactions but with various (reasonable) values of the regularization parameter $R$ can be regarded as an estimator of this type of uncertainty.

In the next section we give a brief description of our method and its numerical realization. The OPE-Gaussian results for the cross section and a comparison of various theoretical uncertainties are shown in Section 3.

\section{2. $3 \mathrm{~N}$ calculations}

The presented results for $\mathrm{Nd}$ scattering have been obtained within the formalism of the Faddeev equations. Its comprehensive description can be found in Refs. 12 and 13 .

In this method the Faddeev equation for an auxiliary state $T|\phi\rangle$ is first solved. In the case the $3 \mathrm{~N}$ force is neglected, it reads

$$
T|\phi\rangle=t P|\phi\rangle+t P G_{0} T|\phi\rangle
$$

where the initial state $|\phi\rangle$ is composed of a deuteron and a relative momentum eigenstate of the projectile nucleon, $P$ is a permutation operator which takes into account the identity of the nucleons and $G_{0}$ is the free $3 \mathrm{~N}$ propagator. The $2 \mathrm{~N}$ interaction $V$ together with the $2 \mathrm{~N}$ free propagator $\tilde{G}_{0}$ appear in the LippmannSchwinger equation for the $2 \mathrm{~N} t$-matrix

$$
t=V+V \tilde{G}_{0} t
$$

We solve Eq. (3) in the momentum space partial wave scheme. We work with the $|p, q, \alpha\rangle$ states with $p=|\vec{p}|$ and $q=|\vec{q}|$ being the magnitudes of the relative Jacobi momenta $\vec{p}$ and $\vec{q}$. Further, $\alpha$ represents a set of discrete quantum numbers for the $3 \mathrm{~N}$ system in the $j I$-coupling ${ }^{12}$. Equation (3) is solved numerically by generating its Neumann series and summing it up by using the Padé method ${ }^{13}$. For results presented here we use all partial waves with $j \leq 5$ and $J \leq \frac{25}{2}$. More details about our numerical performance can be found in Ref. 13 . 


\section{R. Skibiński et al.}

\section{Results}

Knowing the multivariate normal distribution of the OPE-Gaussian potential parameters defined by their expectation values and covariances, it is possible to apply the Monte Carlo statistical approach to estimate related uncertainties for $3 \mathrm{~N}$ observables. Namely, we have repeatedly solved Eq. (3) and calculated observables 51 times taking as an input various sets of potential parameters sampled from their multivariate distribution. The same method has been already used e.g. in Ref. 14 to estimate the uncertainties in question for the ${ }^{4} \mathrm{He}$ bound states and actually we have used the same 50 sampled sets of parameters delivered to us by the members of the Granada group, together with one set with expectation values of parameters.

In Fig. 1 we show the differential cross section $\mathrm{d} \sigma / \mathrm{d} \Omega$ for elastic Nd scattering at two incoming nucleon laboratory energies $E=65$ and $200 \mathrm{MeV}$. At the lower energy predictions based on the AV18 and on the OPE-Gaussian (with the central values of parameters) overlap. Also the red band, which comprises 50 predictions based on the remaining sets of OPE-Gaussian force parameters practically overlaps with previously mentioned predictions. The narrowness of this band clearly shows that at this energy the uncertainty of the cross section arising from the uncertainty of the NN potential parameters is very small for all scattering angles. At the higher energy the spread of the OPE-Gaussian based results becomes somewhat bigger, however, it remains small compared to the difference between predictions based on the AV18 interaction and on the OPE-Gaussian force.

While the work on a systematic comparison of various theoretical uncertainties in $3 \mathrm{~N}$ scattering is under way, in Fig. 2 we take the first step to show the obtained estimators for uncertainties of the differential cross section for elastic $\mathrm{Nd}$ scattering at the incoming nucleon laboratory energy $E=65 \mathrm{MeV}$ and for the scattering
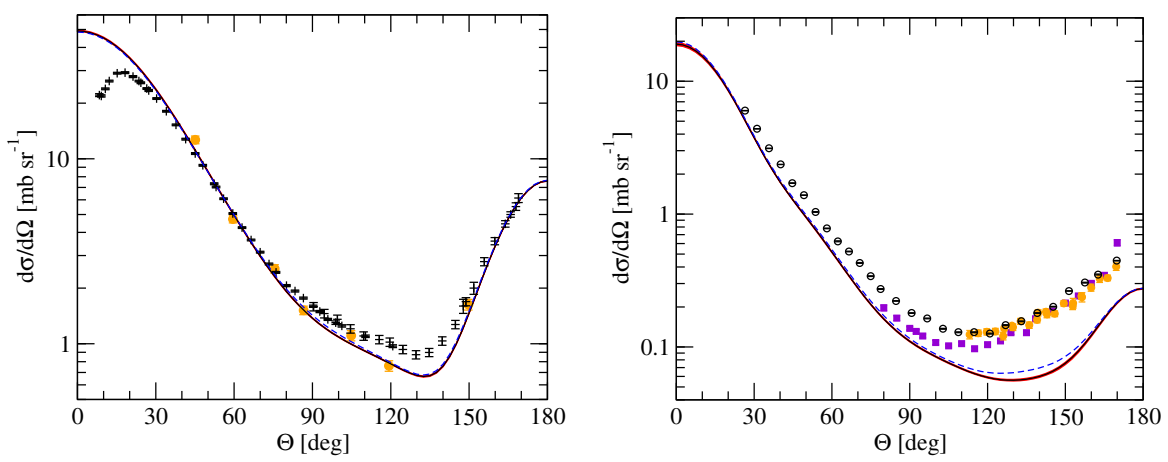

Fig. 1. The differential cross section $\mathrm{d} \sigma / \mathrm{d} \Omega\left[\mathrm{mb} \mathrm{sr}^{-1}\right]$ for elastic Nd scattering at the incoming nucleon laboratory energy $E=65 \mathrm{MeV}$ (left) and $E=200 \mathrm{MeV}$ (right). The black solid (blue dashed) curve represents predictions obtained with the OPE-Gaussian (AV18) force. The red band (practically overlapping with the solid curve) shows the range of predictions obtained with 50 sets of the OPE-Gaussian potential parameters. Data at $E=65 \mathrm{MeV}$ are from Ref. 15 ( $p d$ pluses) and Ref. 16 ( $n d$ orange circles). Data at $E=200 \mathrm{MeV}$ are from Ref. 17 ( $p d$ at $\mathrm{E}=198 \mathrm{MeV}$ violet squares), 18 ( $d p$ at $\mathrm{Ed}=362 \mathrm{MeV}$ orange circles) and 19 ( $p d$ at $\mathrm{E}=190 \mathrm{MeV}$ open circles). 

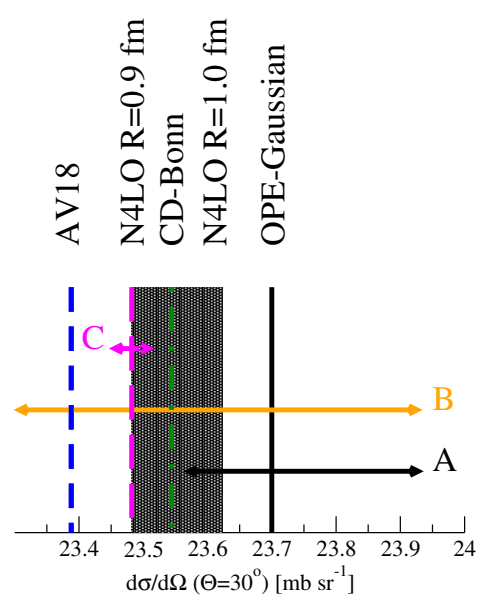

Fig. 2. The values of the differential cross section $\mathrm{d} \sigma / \mathrm{d} \Omega\left[\mathrm{mb} \mathrm{sr}^{-1}\right]$ for elastic Nd scattering at the incoming nucleon laboratory energy $E=65 \mathrm{MeV}$ at the scattering angle $\theta_{c . m} .=30^{\circ}$ and the magnitudes of various theoretical uncertainties for this observable. Vertical blue dashed, magenta long-dashed, green dash-dotted and black solid lines represent predictions obtained with the AV18, chiral $\mathrm{N}^{4} \mathrm{LO}$ with $R=0.9 \mathrm{fm}$, the CD-Bonn and the OPE-Gaussian interactions, respectively. The width of magenta area shows the difference between the chiral $\mathrm{N}^{4} \mathrm{LO}$ predictions for $R=0.9 \mathrm{fm}$ and $R=1.0 \mathrm{fm}$. The horizontal lines A-C show magnitudes of various theoretical uncertainties (see text for details).

angle $\theta_{\text {c.m. }}=30^{\circ}$. The position of a dashed blue, long-dashed magenta, dash-dotted green and solid black segments shows the magnitudes of the cross section obtained with the AV18, chiral N ${ }^{4} \mathrm{LO}$, the CD Bonn and the OPE-Gaussian (with the central values of the parameters), respectively. The width of the light pink area shows the difference between two predictions based on the same chiral $\mathrm{N}^{4} \mathrm{LO}$ interaction but with various values of the regulator, $R=0.9 \mathrm{fm}$ and $R=1.0 \mathrm{fm}$. Note that the values of $R$ in this range are the most recommended as giving the best description of the NN data ${ }^{10,11}$. The horizontal lines show magnitudes of various theoretical uncertainties. The black solid horizontal line (A) shows the range of the predictions obtained with the 50 sets of parameters of the OPE-Gaussian force. The orange horizontal line (B) shows the range of the predictions obtained with the chiral $\mathrm{N}^{4} \mathrm{LO}$ forces for all the five values of the regulator $R(0.8,0.9, \ldots, 1.2 \mathrm{fm})$ used in Ref. 11 . Finally, the magenta horizontal line $(\mathrm{C})$ shows the truncation error $\delta(X)^{(5)}$ (at $\left.\mathrm{N}^{4} \mathrm{LO}\right)$ for the predictions with $R=0.9 \mathrm{fm}$.

For the example shown here the predictions based on various interaction models are relatively close one to another and the distance between them is of the same order as the dominant theoretical uncertainty (determined for a single model) arising from the uncertainty of the NN parameters. We observe that the two uncertainties related to the chiral approach - cut-off dependence (in the range $0.9 \leq R \leq 1.0 \mathrm{fm}$ ) and the truncation errors - amount approximately to $40 \%$ and $15 \%$ of the dominant uncertainty, respectively. Thus, they are not negligible. However, we would like to 


\section{R. Skibiński et al.}

warn the reader that drawing a general conclusion based on only one example can be misleading. Clearly, a more systematic analysis of theoretical uncertainties in $3 \mathrm{~N}$ reactions is required and is being performed.

Summarizing, we confirm that the OPE-Gaussian force belongs to models which can be successfully applied to study three-nucleon reactions. In addition, its properties deliver a great opportunity to estimate theoretical uncertainties in few-body nuclear physics.

\section{Acknowledgments}

We thank Dr. E. Ruiz Arriola and Dr. R. Navarro Pérez for sending us sets of parameters for the OPE-Gaussian model and for the valuable discussions. This work is a part of the LENPIC project and was supported by the Polish National Science Center under Grants No. 2016/22/M/ST2/00173 and 2016/21/D/ST2/01120. The numerical calculations were partially performed on the supercomputer cluster of the JSC, Jülich, Germany.

\section{References}

1. Focus issue Focus on Enhancing the Interaction Between Nuclear Experiment and Theory Through Information and Statistics of J. Phys. G: Nucl. Part. Phys. 42, 030301-034033 (2015), Editors: D.G Ireland and W.Nazarewicz.

2. R.Navarro Pérez, J.E.Amaro, and E.Ruiz Arriola, Phys. Rev. C 88, 064002 (2013); Erratum Phys. Rev. C 91, 029901 (2015).

3. R.Navarro Pérez, J.E.Amaro, and E.Ruiz Arriola, Phys. Rev. C 89, 064006 (2014).

4. R.B.Wiringa, V.G.J.Stoks, and R.Schiavilla, Phys. Rev. C 51, 38 (1995).

5. H.Witała et al., Phys. Rev. C 63, 024007 (2001).

6. J.Kuroś-Żołnierczuk, et al., Phys. Rev. C 66, 024004 (2002).

7. S.Binder et al., Phys. Rev. C 93, 044002 (2016).

8. R.Skibiński et al., Phys. Rev. C 93, 064002 (2016).

9. N.Kalantar-Nayestanaki et al., Rep. Prog. Phys. 75, 016301 (2012).

10. E.Epelbaum, H.Krebs, and Ulf-G.Meißner, Eur. Phys. J. A 51, 26 (2015).

11. E.Epelbaum, H.Krebs, and Ulf-G.Meißner, Phys. Rev. Lett. 115, 122301 (2015).

12. W.Glöckle The Quantum Mechanical Few-Body Problem, Springer-Verlag, Berlin, 1983.

13. W.Glöckle et al., Phys. Rept. 274, 107 (1996).

14. R. Navarro Pérez et al., J. Phys.: Conf. Ser. 742, 012001 (2016).

15. S. Shimizu, et al., Phys. Rev. C 52, 1193 (1995).

16. H. Rühl, et al., Nucl. Phys. A 524, 377 (1991).

17. R.E.Adelberger and C.N.Brown, Phys. Rev. D 5, 2139 (1972).

18. G.Igo et al. Nucl. Phys. A 195, 33 (1972).

19. K.Ermisch et al., Phys. Rev. C 71, 064004 (2005). 